\title{
Os impactos da saúde mental nos estudantes universitários do curso de Enfermagem: revisão bibliográfica
}

\author{
The impacts of mental health on university students in the Nursing course: literature review \\ Los impactos de la salud mental en los estudiantes universitarios del curso de Enfermería: revisión
}

\author{
de la literatura
}

Recebido: 29/11/2021 | Revisado: 03/11/2021 | Aceito: 04/12/2021 | Publicado: 05/12/2021

\author{
Rayane Cristina Canto Martins \\ ORCID: https://orcid.org/0000-0002-0737-1459 \\ Centro Universitário da Amazônia, Brasil \\ E-mail:cristinacanto48@gmail.com \\ Roberta Príscila da Costa Branco \\ ORCID: https://orcid.org/0000-0001-9294-0478 \\ Centro Universitário da Amazônia, Brasil \\ E-mail:roberta-branco@outlook.com
}

\begin{abstract}
Resumo
Introdução: A temática envolvendo a saúde mental tem sido bastante discutida nos últimos anos, assim despertando tal fenômeno para o contexto do Ensino Superior, especificamente aos estudantes universitários do curso de Enfermagem, uma vez que a rotina acadêmica envolvendo estudos de teoria e prática profissional deste é cercada de experiências ambíguos, assim ocasionando a estes acadêmicos altos níveis de estresse e ansiedade. Objetivos: analisar a saúde mental de estudantes universitários do curso de enfermagem por meio de uma revisão bibliográfica, buscando identificar quais as principais doenças mentais enfrentadas pelos estudantes de enfermagem na graduação e discorrer sobre ações de proteção e promoção de saúde mental em estudantes universitários de enfermagem. Métodos: Trata-se de uma pesquisa de natureza qualitativa e descritiva, desenvolvido através de uma revisão integrativa de literatura (RIL), por meio das bases de dados LILACS, MEDLINE, SCIELO, sendo selecionados estudos de 2011 a junho de 2021, com descritores: Saúde Mental; Ansiedade; Estresse; Pandemia Covid-19; Estratégias de saúde; Estudantes Universitários e Enfermagem. Resultados: Dentre os 9 artigos investigados, publicados no ano de 2021, observaramse reações como aumento de estresse, ansiedade, diminuição na qualidade do sono, medo associadas a diminuição do rendimento geralmente por conta da ausência de uma rotina diária e as muitas horas investidas nas mídias sociais/digitais. Conclusão: A saúde mental é um aspecto que deve ser tratado com um dos pilares para o processo de ensino-aprendizagem dos estudantes do curso de enfermagem, visto que seu papel de cuidar é essencial manter-se sempre em base de equilíbrio.
\end{abstract}

Palavras-chave: Saúde mental; Ansiedade; Estresse; Estudantes universitários; Enfermagem.

\begin{abstract}
Introduction: The theme involving mental health has been widely discussed in recent years, thus awakening this phenomenon to the context of Higher Education, specifically to university students of the nursing course, since the academic routine involving studies of theory and professional practice of this is surrounded by ambiguous experiences, thus causing these students high levels of stress and anxiety. Objectives: to smooth the mental health of university students of the nursing course through a literature review, seeking to identify the main mental illnesses faced by nursing students in undergraduate studies and discuss actions to protect and promote mental health in nursing university students. Methods: This is a qualitative and descriptive research, developed through an integrative literature review (ILR), through lilacs, MEDLINE, SCIELO databases, and studies from 2011 to June 2021 were selected, with descriptors: Mental Health; Anxiety; Stress; Pandemic Covid-19; Health strategies; University Students and Nursing. Results: Among the 9 articles investigated, published in 2021, reactions such as increased stress, anxiety, decreased sleep quality, fear associated with decreased performance were generally due to the absence of a daily routine and the many hours invested in social/digital media. Conclusion: Mental health is an aspect that should be treated as one of the pillars for the teaching-learning process of nursing students, since their role of caring is essential to always remain on a balance basis.
\end{abstract}

Keywords: Mental health; Anxiety; Stress; College students; Nursing.

\section{Resumen}

Introducción: El tema que involucra la salud mental ha sido ampliamente discutido en los últimos años, despertando así este fenómeno al contexto de la Educación Superior, específicamente a los estudiantes universitarios de la carrera de Enfermería, ya que la rutina académica involucra estudios de teoría y práctica profesional de esta es rodeado de 
experiencias ambiguas, provocando así en estos académicos altos niveles de estrés y ansiedad. Objetivos: analizar la salud mental de los estudiantes universitarios de enfermería a través de una revisión de la literatura, buscando identificar las principales enfermedades mentales que enfrentan los estudiantes de licenciatura en enfermería y discutir acciones para proteger y promover la salud mental en los estudiantes universitarios de enfermería. Métodos: Se trata de una investigación cualitativa y descriptiva, desarrollada a través de una revisión integradora de la literatura (RIL), a través de las bases de datos LILACS, MEDLINE, SCIELO, con estudios seleccionados desde 2011 hasta junio de 2021, con descriptores: Salud mental; Ansiedad; Destaca; Pandemia de COVID-19; Estrategias de salud; Universitario y Enfermería Estudiantes. Resultados: Entre los 9 artículos investigados, publicados en 2021, hubo reacciones como aumento del estrés, ansiedad, disminución de la calidad del sueño, miedo asociado a disminución del rendimiento, generalmente por ausencia de una rutina diaria y muchas horas invertidas en social / digital. medios de comunicación. Conclusión: La salud mental es un aspecto que debe ser tratado como uno de los pilares del proceso de enseñanza-aprendizaje de los estudiantes de enfermería, y su papel de cuidado es fundamental para mantener siempre una base equilibrada.

Palabras clave: Salud mental; Ansiedad; Estrés; Estudiantes universitarios; Enfermería.

\section{Introdução}

Os termos envolvendo saúde e saúde mental apresentam conceitos complexos e oriundos de contextos sócio-políticos e pela evolução de práticas em saúde. Segundo a Organização Mundial de Saúde (OMS), defini a saúde como um estado de completo bem-estar físico, mental e social, e não consiste somente na ausência de doença ou então de alguma enfermidade existente (OMS, 2015).

Enquanto que a respeito da saúde mental, a OMS conceitua como sendo um estado de bem-estar no qual o sujeito percebe suas próprias habilidades, em poder lidar com os estresses cotidianos, assim como podendo trabalhar produtivamente e é capaz de contribuir para sua comunidade (OMS, 2015).

Na tentativa de abarca esses dois conceitos, o Sistema Único de Saúde brasileiro (SUS) ao longo do tempo, vem conseguindo adotar um conceito ampliado de saúde, que inclui em suas prioridades o cuidado à saúde mental (Fertonani et al., 2015).

Diante da temática da saúde mental, nota-se que no contexto do ensino superior, diversas situações ocorridas no cotidiano dos universitários requer um processo constante de adaptação, que influencia diretamente no seu processo de amadurecimento, além do seu desenvolvimento cognitivo e afetivo. Contudo, quando determinadas situações estão associadas as vulnerabilidades emocionais e sociais, podem levar ao sofrimento psíquico, que juntamente com as demandas oriundas da universidade acabam por desencadear ou até mesmo agravar esses problemas de saúde física e mental (Lacerda, 2016).

Na população universitária tem sido bastante comum a apresentação de sintomas psicopatológicos em decorrência de situações vivenciadas a partir de sua entrada no ensino superior, assim torna-se recorrente casos de estudantes que apresentam algum tipo de sintomatologia psicopatológica ao longo de sua formação, como no caso de depressão e ansiedade (Maia \& Dias, 2020).

Em cursos da área da saúde, esses casos são ainda mais comuns, onde esses estudantes apresentam altos níveis de estresse e ansiedade, por este fato, é de fundamental importância, ter um olhar cuidadoso acerca da vulnerabilidade dessa população em relação ao uso de substâncias psicoativas (Bolsonisilva \& Loureiro, 2016).

Deste modo, cursos da área da saúde, como enfermagem, por mobilizarem sentimentos em relação à futura atuação, além de trabalhar diretamente com o zelo pela vida humana, pode ocasionar de certa forma maior exposição do aluno ao estresse diante da responsabilidade do cuidado, da prestação de um atendimento adequado, além de saber lidar emocionalmente com o sofrimento muitas vezes expresso pelas pessoas que buscam os serviços de saúde (Silva et al., 2018).

A temática desta pesquisa se apresenta relevante, visto que cada vez mais, a saúde mental no ambiente universitário e entre estudantes da área de saúde vem sendo debatido, com o aparecimento constante de relatos de acadêmicos que sofrem com diagnósticos de ansiedade, depressão e dentre outros transtornos, o que faz refletir que essas questões ressaltam a importância da ampliação de estratégias para prevenção e promoção de saúde mental de estudantes no contexto universitário. 
Diante do fenômeno analisado, procurou-se compreender a seguinte problemática: De que forma a ausência de saúde mental pode interferir na vivência do estudante universitário?

Portanto o objetivo geral da pesquisa foi analisar a saúde mental dos estudantes universitários do curso de enfermagem por meio de uma revisão bibliográfica, e específicos identificar quais as principais doenças mentais enfrentadas pelos estudantes de enfermagem na graduação, e conhecer ações de proteção e promoção da saúde mental em estudantes Universitários de enfermagem.

O conhecimento sobre a saúde mental dos estudantes universitários do curso de enfermagem contribuirá para o desenvolvimento de novas técnicas e instrumentos mais eficazes a serem trabalhados dentro do contexto universitário.

\section{Metodologia}

Este é um estudo de natureza qualitativa e descritiva, desenvolvido através de uma pesquisa bibliográfica e uma Revisão Integrativa de Literatura (RIL). A revisão integrativa é um método específico, que resume o passado da literatura empírica ou teórica, para fornecer uma compreensão mais abrangente de um fenômeno particular (Souza et al., 2010).

Foram selecionados artigos com relação ao objeto de estudo, referentes ao período de 2011 a junho de 2021, sendo incluídos artigos de publicação nacional e internacional, e que tenham como descritores: Saúde Mental; Ansiedade; Estresse; Estratégias de saúde; Estudantes Universitários e Enfermagem.

Buscou-se acervos nas bases de dados da Literatura Latino-americana e do Caribe em Ciências da Saúde (LILACS), Medical Literature Analysisand Retrieval Sistem (MEDLINE), Pubmed, Scientific Electronic Library Online (SCIELO). As buscas foram guiadas por descritores em ciências da saúde (DeCS).

Para análise foi utilizada a "Análise de Conteúdo Temático de Bardin”, cujo objetivo é compreender o sentido das comunicações e suas significações explícitas e/ou ocultas (BARDIN, 2006).

Desse modo, as análises do estudo foram apresentadas na seguinte sequência: descrição dos dados de identificação das publicações (autores e ano), do Estado e Instituição sede do estudo e do tipo de revista científica; e a seguir organizados em um quadro, que descreveu as características metodológicas dos estudos, classificando-os de acordo com o delineamento de pesquisa e nível de evidências; descrição da análise propriamente dita, agrupada em categorias.

Foram encontrados 99 artigos nas plataformas escolhidas dos quais utilizou-se 09 artigos para elaboração da pesquisa, que apresentaram os seguintes critérios: artigos completos disponíveis em língua portuguesa e inglesa que abordaram a temática escolhida e que estivessem no período definido para o estudo (Fluxgrama 1). Como critérios de exclusão optou-se por não utilizar textos incompletos e publicações fora do período definido no estudo. 
Figura 1- Fluxograma dos artigos encontrados.

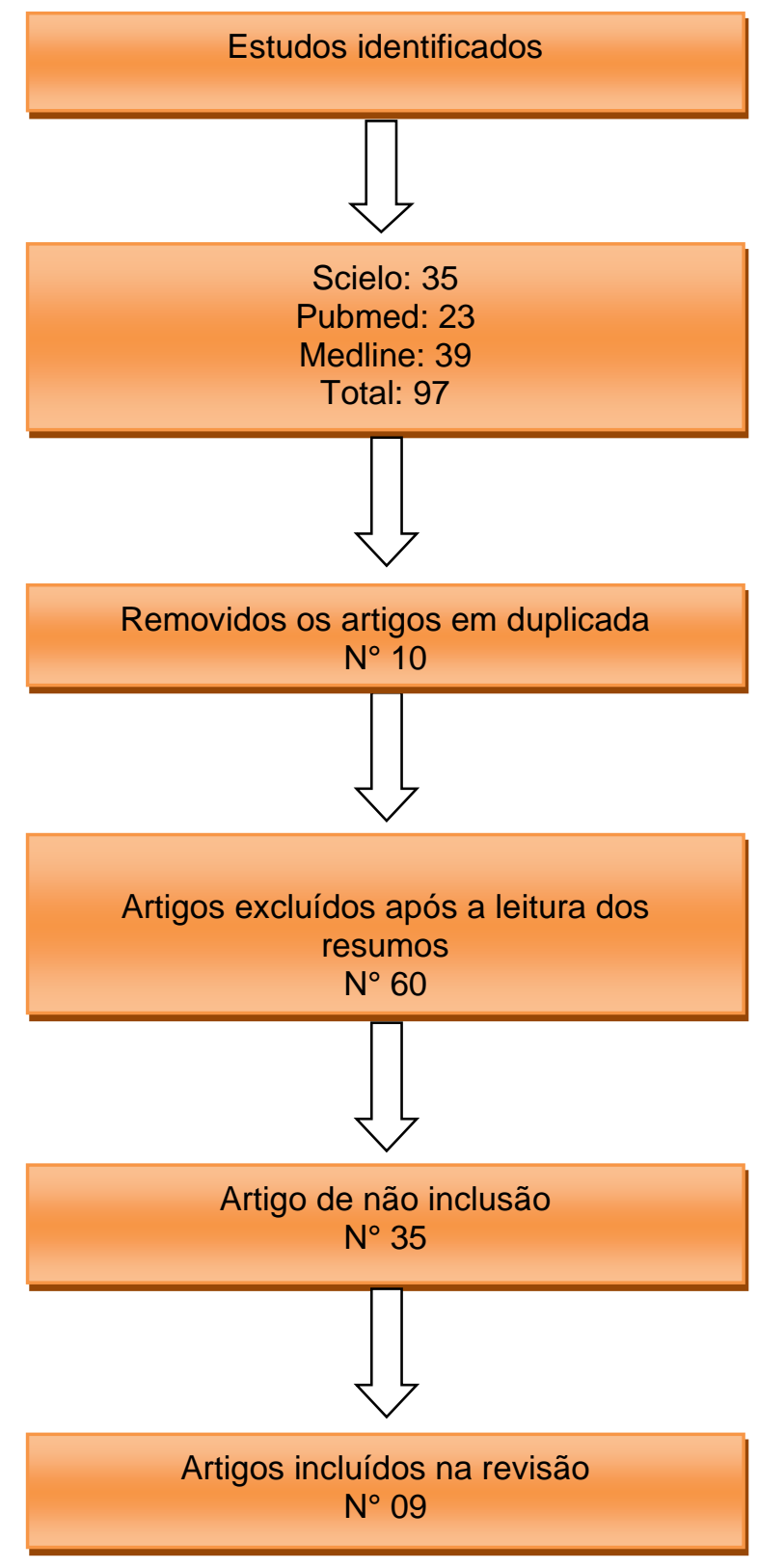

Fonte: Autores (2021)

Os artigos selecionados para síntese foram estruturados em um quadro criado com o auxílio do software Microsoft Word 365 com a seguinte disposição: $1^{\mathrm{a}}$ coluna título, autores, ano de publicação; $2^{\mathrm{a}}$ coluna objetivo; $3^{\mathrm{a}}$ coluna resultados, em sequência foi realizada a discussão com base nos artigos selecionados.

\section{Resultados}

No intuito de contribuir nos resultados da pesquisa, foi desenvolvido um quadro (Quadro 1) com as principais informações relacionadas ao tema desta revisão, no intuito de proporcionar ao leitor uma compreensão melhor acerca dos fatos pesquisados, conforme demonstrado detalhadamente no Quadro 1 a seguir. 
Quadro 1: Síntese com informações dos artigos selecionados.

\begin{tabular}{|c|c|c|}
\hline Título / Autores/ Ano & Objetivo & Resultado \\
\hline $\begin{array}{l}\text { Saúde mental de estudantes } \\
\text { universitários durante a } \\
\text { pandemia de Covid-19. } \\
\text { GUNDIM et al. (2021) }\end{array}$ & $\begin{array}{l}\text { Identificar as evidências disponíveis } \\
\text { sobre formas de apresentação do } \\
\text { sofrimento psíquico e ações de proteção e } \\
\text { promoção da saúde mental em estudantes } \\
\text { universitários, durante a pandemia de } \\
\text { COVID-19. }\end{array}$ & $\begin{array}{l}\text { Dentre os } 7 \text { artigos investigados, publicados em } \\
\text { 2020, observaram-se reações emocionais descritas } \\
\text { por estresse, ansiedade, luto, raiva e pânico, } \\
\text { associadas à preocupação com o atraso das } \\
\text { atividades acadêmicas e ao medo de adoecer. }\end{array}$ \\
\hline 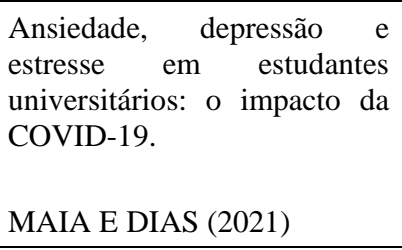 & $\begin{array}{l}\text { Analisar se os níveis de depressão, } \\
\text { ansiedade e estresse em estudantes } \\
\text { universitários se alteraram no período } \\
\text { pandêmico (2020) comparativamente a } \\
\text { períodos anteriores/normais. }\end{array}$ & $\begin{array}{l}\text { Os resultados sugerem um impacto psicológico } \\
\text { negativo da pandemia nos estudantes. Importa } \\
\text { continuar a explorar as implicações da pandemia na } \\
\text { saúde mental dos estudantes, para que se possam } \\
\text { prevenir e minorar os seus efeitos. }\end{array}$ \\
\hline $\begin{array}{l}\text { Associação entre bem-estar } \\
\text { pessoal, sociodemográficos e } \\
\text { antropométricos em } \\
\text { estudantes universitários. } \\
\text { SILVA JUNIOR et al. (2021) }\end{array}$ & $\begin{array}{l}\text { Analisar a existência de associação entre } \\
\text { bem-estar pessoal e variáveis } \\
\text { sociodemográficas e antropométricas em } \\
\text { universitários. }\end{array}$ & $\begin{array}{l}\text { O perfil encontrado na maioria de universitários foi } \\
\text { sexo feminino, adultos jovens, solteiros, que não } \\
\text { possuem outra graduação, pardos e com renda } \\
\text { familiar de até um salário mínimo e através de } \\
\text { alguns indicadores mostrou baixo comportamento } \\
\text { de promoção da saúde e insatisfação com o bem- } \\
\text { estar pessoal nos universitários. }\end{array}$ \\
\hline $\begin{array}{l}\text { Índices de depressão, } \\
\text { ansiedade e estresse entre } \\
\text { estudantes de enfermagem e } \\
\text { medicina do Acre. } \\
\text { SANTIAGO et al. (2021) }\end{array}$ & $\begin{array}{l}\text { Evidenciar índices de depressão, } \\
\text { ansiedade e estresse entre estudantes dos } \\
\text { cursos de enfermagem e medicina de um } \\
\text { centro universitário do Acre. }\end{array}$ & $\begin{array}{l}\text { A saúde mental dos estudantes pode encontrar-se } \\
\text { em risco, o que evidencia a necessidade de } \\
\text { implementação de um projeto político-pedagógico } \\
\text { pela instituição de ensino, voltado para essa } \\
\text { temática, apresentando estratégias para o } \\
\text { enfrentamento dessa realidade, que vise o bem- } \\
\text { estar dessa população. }\end{array}$ \\
\hline $\begin{array}{l}\text { Ansiedade: fatores } \\
\text { predisponentes em estudantes } \\
\text { universitários para o } \\
\text { adoecimento mental. } \\
\text { GALVÃO et al. (2021) }\end{array}$ & $\begin{array}{l}\text { Investigar o perfil de estudantes } \\
\text { universitários que possuem ansiedade e } \\
\text { avaliar os fatores predisponentes para o } \\
\text { adoecimento mental }\end{array}$ & $\begin{array}{l}\text { Entre os estudantes com algum nível de ansiedade, } \\
\text { a maioria apresentou ansiedade leve }(25,1 \%) \text {, } \\
\text { seguido de elevada }(22,9 \%) \text { e moderada }(16,3 \%) \text {, } \\
\text { sendo observada no sexo feminino }(43,3 \%) \text {. }\end{array}$ \\
\hline $\begin{array}{l}\text { Sentimentos desenvolvidos } \\
\text { pelos alunos do último ano do } \\
\text { curso de enfermagem } \\
\text { relacionado à COVID-19. } \\
\text { SALIN et al. (2021) }\end{array}$ & $\begin{array}{l}\text { Descrever os sentimentos desenvolvidos } \\
\text { pelos alunos do último ano do curso de } \\
\text { enfermagem relacionado à COVID-19 em } \\
\text { uma Instituição de Ensino Superior no } \\
\text { município de Porto Velho - RO. }\end{array}$ & $\begin{array}{l}\text { A pesquisa apontou que os estudantes } \\
\text { desenvolveram sentimentos ruins como: medo, } \\
\text { ansiedade, angústia, desespero, tristeza e solidão. } \\
\text { Mas também sentimentos bons, solidariedade, } \\
\text { esperança e otimismo. }\end{array}$ \\
\hline $\begin{array}{l}\text { Sintomas de depressão em } \\
\text { estudantes de enfermagem. } \\
\text { SOUSA et al. (2021) }\end{array}$ & $\begin{array}{l}\text { Verificar sintomas de depressão entre os } \\
\text { estudantes do curso de enfermagem em } \\
\text { uma universidade privada. }\end{array}$ & $\begin{array}{l}\text { A análise dos resultados demonstrou que os } \\
\text { sintomas depressivos estacam presentes em } 45,56 \% \\
\text { da amostra. Os resultados contribuem para análise } \\
\text { da saúde mental dos acadêmicos do curso de } \\
\text { enfermagem, e a necessidade da promoção do bem- } \\
\text { estar psicológico destes estudantes. }\end{array}$ \\
\hline $\begin{array}{l}\text { A escuta ativa no processo de } \\
\text { ensino-aprendizagem dos } \\
\text { acadêmicos de enfermagem. }\end{array}$ & $\begin{array}{l}\text { O objetivo deste trabalho é descrever as } \\
\text { ações referentes à escuta ativo-terapêutica } \\
\text { de discentes do curso de enfermagem e } \\
\text { analisar as repercussões desta escuta no } \\
\text { processo de ensino-aprendizagem } \\
\text { utilizando como estratégia as plataformas } \\
\text { de educação à distância. }\end{array}$ & $\begin{array}{l}\text { A escuta ativa permitiu aos discentes falaram sobre } \\
\text { suas emoções e sentimentos experiências durante a } \\
\text { Pandemia. Também estimulou aprendizagem de } \\
\text { forma ativa, despertando o pensamento crítico dos } \\
\text { estudantes, o autoconhecimento, o desenvolvimento } \\
\text { e a aquisição de habilidades pautadas no agir, no } \\
\text { ouvir e no sentir. }\end{array}$ \\
\hline $\begin{array}{l}\text { Saúde mental e qualidade do } \\
\text { sono entre estudantes } \\
\text { universitários em tempos de } \\
\text { pandemia da COVID-19: } \\
\text { experiência de um programa } \\
\text { de assistência estudantil. }\end{array}$ & $\begin{array}{l}\text { Apresentar a experiência de um grupo de } \\
\text { professores universitários e uma monitora } \\
\text { na construção, implementação e } \\
\text { consolidação de um projeto de } \\
\text { intervenção sobre a saúde mental e } \\
\text { qualidade do sono, em tempos de } \\
\text { pandemia da COVID - 19, entre alunos de } \\
\text { graduação de uma universidade pública } \\
\text { do Estado da Bahia. }\end{array}$ & $\begin{array}{l}\text { Os alunos relataram suas vivências e seus } \\
\text { sentimentos diante de tal situação que assolou o } \\
\text { Brasil e o mundo, como mudança de rotina, } \\
\text { alterações do sono, desânimo para realizar } \\
\text { atividades, falta do ambiente acadêmico e dos } \\
\text { colegas, questões relacionadas ao produtivismo, } \\
\text { receio pelos familiares grupo de risco, dentre outros }\end{array}$ \\
\hline
\end{tabular}

Fonte: Martins (2021), a partir das bases de dados. 


\section{Discussão}

Por meio das análises dos artigos foi possível analisar os seguintes fatores: A saúde mental nos estudantes universitários do curso de enfermagem; as principais doenças mentais enfrentadas pelos estudantes de enfermagem na graduação, e ações de proteção e promoção da saúde mental em estudantes Universitários de enfermagem.

No que se refere a saúde mental dos estudantes universitários do curso de enfermagem, Bublitz et al. (2016) acredita que a partir do momento que esses indivíduos ingressam para o ambiente universitário podem vivenciar situações de estresse até conseguiram de fato se adaptar a essa nova condição, com novas obrigações e responsabilidades, podendo assim apresentar sintomas de depressão e ansiedade, devido à pressão por bom desempenho acadêmico, e o medo do novo, deixando-os mais vulneráveis psicologicamente.

Na perspectiva de Moura (2019) tal fato pode ocorrer em virtude de o indivíduo vivenciar comportamentos diferenciados, quando se trata do relacionamento social, acarretando problemas futuros relacionados à sua vida profissional.

De acordo com Salin et al. (2021), em seu estudo buscou conhecer os sentimentos desenvolvidos pelos alunos do último ano do curso de enfermagem relacionado à COVID-19 em uma Instituição de Ensino Superior no município de Porto Velho - RO, concluindo que sentimentos como: preocupação, tristeza, solidão, estresse, medo, sobrecarga, desmotivação, além de solidariedade, esperança e otimismo, foram desenvolvidos em consequência da Pandemia de Covid-19.

O autor supracitado afirma que a suspensão das atividades didáticas presenciais, e o redirecionamento ao ensino remoto, como medida de prevenção disponível e assegurada as necessárias condições que promovam um ensino de qualidade no contexto a Covid-19 contribui para tais sentimentos externalizados pelo público pesquisado.

Silva et al. (2020), explique que o ensino à distância promove desafios, para o qual alguns docentes e discentes, ainda não se encontram preparados, o que pode interferir no processo de ensino-aprendizagem no desenvolvimento de sentimentos como insegurança e incertezas.

Sousa (2021) apud Padovani (2014, p.3), faz a seguinte afirmação:

Os estudantes universitários fazem parte de uma população propensa a desenvolver sintomas depressivos, mesmo que seja em grau leve, devido aos fatores estressantes vivenciados dentro da graduação (sobrecarga de informação, exigência dos professores, novas adaptações a graduação, escassez de tempo para o lazer, fatores estressantes familiares, limitação de finanças,), esses fatores podem desencadear transtornos mentais. Entanto essa vivência dentro da graduação, exige maior desempenho do estudante.

No que diz respeito as principais doenças mentais enfrentadas pelos estudantes de enfermagem na graduação, Sousa et al. (2021), constatou sintomas de depressão entre os estudantes do curso de enfermagem em uma Universidade privada, identificando que o grupo de estudantes do sexo feminino, teve maiores sintomas depressivos, sendo maior público dentro da graduação em enfermagem. Porém, vale frisar que os sintomas depressivos apresentados por acadêmicos de enfermagem não devem ser alvo de descriminação, e sim de necessidade de atenção ao cuidado, e elaboração de estratégias para que seja possível ocorrer a diminuição dos sintomas dentro do curso em questão (Padovani et al., 2015).

Nos estudos de Maia \& Dias (2021), foram analisados os níveis de depressão, ansiedade e estresse em estudantes universitários no período pandêmico (2020) em comparação aos períodos anteriores. Os resultados demonstraram que houve um aumento significativo de ansiedade, depressão e estresse entre os estudantes universitários em relação nesse tempo de pandemia, comparado aos períodos não pandêmicos.

O resultado do estudo realizado pelos autores acima citados, teve como objetivo explorar os níveis de ansiedade, depressão e estresse em estudantes universitários, comparando dois momentos distintos, isto é, um período normal e o período pandêmico afirmando que: 
Ocorreram aumentos significativos de perturbação psicológica (ansiedade, depressão e estresse) entre os estudantes universitários no período pandêmico comparativamente a períodos normais. Esses resultados vão ao encontro de outros estudos internacionais que analisaram o efeito psicológico da COVID-19 e de outras pandemias (Maia \& Dias, 2021, p.5).

O estudo aponta que a pandemia provocou efeitos que são capazes de prejudicar demasiadamente a saúde mental dos universitários, tornando-se importante descrever os sentimentos desenvolvidos pelos alunos do último ano do curso de enfermagem.

Santiago et al. (2021), em seu estudo constatou o aumento nos índices de depressão, ansiedade e estresse entre estudantes dos cursos de enfermagem e medicina de um centro universitário do Acre. Conforme os relatos apontados pelo autor em sua pesquisa, identificou que ao tratar-se de estresse, os acadêmicos de ambos os cursos, apresentaram resultados semelhantes, especialmente no início da vida acadêmica.

No que se refere as ações de proteção e promoção da saúde mental em estudantes Universitários de enfermagem, Gundim et al. (2021), realizou um estudo objetivando a identificação de evidenciais sobre formas de apresentação do sofrimento psíquico e ações de proteção e promoção da saúde mental em universitários, durante o período de emergência de saúde pública, a autora entendeu a necessidade de identificar tais medidas de prevenção e promoção de saúde mental, pois com a mudança repentina que certas medidas impostas à população de maneira inesperada que resultou em uma reorganização social abrupta, trouxe consigo diversas consequências que refletiu na população em geral e nos universitários.

Em relação as ações de proteção e promoção da saúde mental voltada para estudantes universitários, a autora afirma em sua obra que:

Os sistemas de saúde e as autoridades governamentais de saúde mental de estudantes universitários durante a pandemia da COVID-19 devem estar preparados para as transmissões generalizadas de doenças, bem como para todas as consequências possíveis, incluindo os impactos psicológicos a que esses indivíduos estão submetidos diante uma situação de epidemia. Frente ao período de emergência de saúde pública, entende-se a necessidade de identificar medidas de prevenção e promoção de saúde mental desses estudantes (Gundim, 2021 apud Araújo, 2020, p.6).

Coelho et al. (2021), apresenta a "roda de conversas online" como proposta de ampliar os vínculos com o público discente, evitando o isolamento social ainda maior do que o existente, neste período e, assim, promover a saúde mental e qualidade do sono, reduzindo o risco de sofrimento psíquico, bem como o agravamento de transtornos mentais, como ansiedade e depressão, o resultado, concluiu que:

A partir das falas dos estudantes foi possível identificar alterações na rotina, fato que já era de se esperar devido às mudanças decorrentes da pandemia. Observou-se, sobretudo, um destaque para dificuldades em realizar tarefas do cotidiano, que estavam relacionadas ao desânimo em implementá-las[...] Entre os relatos que emergiram durante as "rodas" de conversa, o sono foi pontuado pela maioria dos participantes como algo prejudicado nesse período de pandemia se que os discentes relataram com uma maior frequência os impasses em vivenciar a quarentena devido à ausência da rotina que possuíam antes da pandemia, na qual incluía o contato frequente com familiares, amigos e colegas da universidade. Foi notório também a predominância de relatos relacionados ao surgimento de sentimentos como a ansiedade, o medo, a preocupação e a impotência devido o isolamento social apresentar-se como uma situação nova e desconhecida. No que tange a qualidade de sono a ausência de uma rotina diária e as muitas horas investidas nas mídias sociais/digitais foram referidas como causadoras de uma irregularidade significativa no sono/repouso (Coelho, 2021, p.3).

Outra estratégia de ação de promoção e proteção da saúde mental dos estudantes foi a aplicação de questionários específicos para os cursos de graduação e pós-graduação como mecanismo para identificar as diferentes formas de sofrimento, e dessa forma realizar uma classificação de acordo com sua gravidade e risco, e posteriormente traçar um plano de cuidados para minimizar ou cessar esse sofrimento no ambiente acadêmico, bem como, criar encontros para práticas integrativas e 
complementares (aromaterapia, ioga, meditação, entre outras) para diminuição do estresse (Gundim, 2021 apud Teixeira, 2020).

Entende-se que o apoio social reduz a pressão psicológica e torna-se eficaz e necessário durante emergências de saúde pública, por isso que os profissionais de saúde precisam identificar as necessidades psicológicas desses estudantes, inclusive de profissionais especializados que pretende mitigar o sofrimento emocional e diminuir os possíveis agravantes (Gundim et al. 2021).

Portanto, as Universidades, profissionais da saúde, e autoridades da saúde, devem exercer um papel fundamental nesse campo, a partir do momento que elaboram diretrizes e ações de enfrentamento ao adoecimento psíquico, possibilitando a diminuição de casos mais graves com vistas à promoção da saúde dos universitários.

\section{Conclusão}

A presente pesquisa buscou analisar a saúde mental dos estudantes universitários do curso de enfermagem por meio de uma revisão da literatura, a partir dos dados obtidos através dos artigos selecionados e publicados no período de 2011 a junho de 2021.

Os resultados obtidos foram considerados satisfatórios para a pesquisa. O presente estudo apontou aspectos referentes a saúde mental de estudantes universitários, principais doenças mentais apresentadas por este público e ações propostas para promoção e prevenção da saúde mental dos acadêmicos.

A partir da análise realizada, concluiu-se que a saúde mental é um aspecto que deve ser tratado com um dos pilares para o processo de ensino-aprendizagem dos estudantes do curso de enfermagem, visto que seu papel de cuidar é essencial manter-se sempre em base de equilíbrio.

Em relação as principais doenças mentais, a pesquisa apontou que as mais recorrentes são ansiedade, depressão e estresse. No que se refere as ações de promoção e prevenção da saúde mental concluiu-se que atividades como: roda de conversas online para realização de escuta, encontros para práticas integrativas e complementares de aromaterapia, ioga e meditação para diminuição do estresse, além de aplicação de questionários específicos para acadêmicos, com intuito de identificar o sofrimento psíquico e propor um tratamento eficaz de acordo com a realidade identificada.

No decorrer dos estudos foi possível identificar o fenômeno da COVID-19 em várias pesquisas, como fator que interferiu na vida acadêmica e na saúde física dos estudantes e de seus familiares causando efeitos negativos à saúde mental dos universitários de enfermagem.

Este estudo mostra-se relevante acima de tudo em três âmbitos: no âmbito científico ao cooperar com insumos teóricos e metodológicos para novos conhecimentos que possam ser gerados futuros, no âmbito acadêmicos ao enriquecer com aprendizado da sociedade acadêmica, e por fim no âmbito social, pois permite uma discussão em torno de um tema que merece tanta atenção em nossa sociedade.

Diante do exposto entende-se a importância da inserção da temática da saúde mental no ambiente universitário, além de adoção de estratégias de redução, prevenção e manejo do sofrimento psíquico, tanto por parte das autoridades de saúde quanto pelas universidades e profissionais da educação, para que seja possível minimizar o sofrimento emocional, bem como os seus efeitos futuros aqueles que apresentam diagnósticos ou predisposição aos transtornos mentais decorrentes da ausência de saúde mental.

Além disso, entende-se a importância do desenvolvimento de artigos e/ ou trabalhos futuros para a discussão de novos dados, para que assim, profissionais da área da saúde bem como a sociedade como todos, tenho conhecimento atualizado sobre a temática. 
Research, Society and Development, v. 10, n. 16, e319101624079, 2021

(CC BY 4.0) | ISSN 2525-3409 | DOI: http://dx.doi.org/10.33448/rsd-v10i16.24079

\section{Referências}

Araújo, F. J. O., Lima, L. S. A., Cidade, P. I. M., Nobre, C. B. \& Rolim, Neto., M. L. (2020). Impact of Sars-Cov-2 And Its Reverberation in Global Higher Education and Mental Health. Psychiatry Res. V.5, n.2, p.13-21.

Bardin, L. (2006). Análise de conteúdo. Lisboa: Edições 70.

Bolsoni-Silva, A. T. \& Loureiro, S. R. (2016). O Impacto das Habilidades Sociais para a Depressão em Estudantes Universitários. Psic.: Teor. e Pesq, vol.32, n.4, 200-210.

Bublitz, S., Guido, L. A., Lopes, L. F. D. \& Freitas, E. O. Associação entre estresse e características sociodemográficas e acadêmicas de estudantes de enfermagem. Texto \& Contexto-Enfermagem, 2016; 25(4), 120-130.

Coelho, E. et al. (2021). Saúde mental docente e intervenções da Psicologia durante a pandemia. PSI UNISC, 5(2), 20-32. doi: 10.17058/psiunisc.v5i2.16458

Fertonani, H. P., Pires, D. E.P., Biff, D. \& Scherer, M. D. A. (2015). The health care model: concepts and challenges for primary health care in Brazil. Ciênc Saúde Coletiva, Jun;20(6):1869-78.

Gundim, V. A., Encarnação, J. P., Santos, F. C., Santos, J. E., Vasconcellos, E. A. \& Souza, R. C. (2021). Saúde mental de estudantes universitários durante a pandemia de COVID-19. Rev baiana enferm, v.35, n.10, p.39-49.

Maia, B. R. \& Dias, P. C. (2020). Ansiedade, depressão e estresse em estudantes universitários: o impacto da COVID-19. Estud psicol (Campinas), v.37, n.12, p.10-16.

Moura, J. B. et al. (2019). Ansiedade em acadêmicos dos cursos da área da saúde de uma universidade privada. https://www.univale.br/wpcontent/uploads/2019/12/enfer.-2019_2-ansiedade-macad\%c3\%8amicos-dos-cursos-da-\%c3\%81rea-da-sa\%c3\%9ade-de-uma-universidade-privada...-juliana.kristyanne.-let\%c3\%8dcia.-mila.pdf.

Padovani, R. C. et al. (2014). Vulnerabilidade e bem-estar psicológicos do estudante universitário. Revista brasileira de terapias cognitivas, v. 10, n. 1, p. 0210. http://pepsic.bvsalud.org/scielo.php?script=sci_arttext\&pid=S180856872014000100002.

Rodrigues, S. B.; Rodrigues, D. S. \& Carvalho, R. D. (2020). Campos Panorama da saúde mental dos estudantes de enfermagem: Uma revisão integrativa de literatura. Braz. J. Hea. Rev., Curitiba, v. 3, n. 3, p.5928-5942 may./jun.

Salin, A. B. et al. (2021). Sentimentos desenvolvidos pelos alunos do último ano do curso de enfermagem relacionado à covid-19. COVID-19: O Trabalho dos Profissionais da Saúde em Tempos de Pandemia, v.5, n.2; p. 192-212.

Santiago, M.B., Braga, OS., Silva, PR., Capelli, VMR., \& Costa, RSL. (2021). Índices de depressão, ansiedade e estresse entre estudantes de enfermagem e medicina do Acre. Revista Psicologia, Diversidade e Saúde, 10(1), 73-84. http://dx.doi. org/10.17267/2317-3394rpds.v10i1.3374

Silva, C. P., Souza, F. A. D., Albuquerque, F. D. N., Almeida, T. A. \& Araújo, L. F. D. (2018). Psicologia e saúde mental: um estudo das representações entre universitários de psicologia. Salud Soc, 9(3):210-20.

Silva, T. M. O. et al. Conceitos dos discentes de enfermagem sobre aulas remotas. Revista Diálogos em Saúde, 2020, Sao Paulo, v. 3, n. 1, p. 47-61.

Sousa, T. B., Romeiro, M. A. S. \& Sandim, L. C. Sintomas de depressão em estudantes de enfermagem. Revista Científica Multidisciplinar Núcleo do Conhecimento. Vol. 02, n.1, pp. 78-88. 